\title{
Up-regulated LncRNA-ATB regulates the growth and metastasis of cholangiocarcinoma via miR-200c signals
}

This article was published in the following Dove Press journal: OncoTargets and Therapy

\author{
Hai Lin ${ }^{1,2}$ \\ Lili Yang ${ }^{2}$ \\ Feng Tian $^{2}$ \\ Shuang $\mathrm{Nie}^{3}$ \\ Hailang Zhou ${ }^{4}$ \\ Jun Liu ${ }^{5}$ \\ Weichang Chen' \\ 'Department of Gastroenterology, The \\ First Affiliated Hospital of Soochow \\ University, Suzhou, Jiangsu 215006, \\ People's Republic of China; ${ }^{2}$ Department \\ of Gastroenterology, Lin Yi Central \\ Hospital, Linyi, Shandong 276400, \\ People's Republic of China; ${ }^{3}$ Department \\ of Gastroenterology, Drum Tower \\ Hospital, Medical School of Nanjing \\ University, Nanjing, Jiangsu 210008, \\ People's Republic of China; ${ }^{4}$ Department \\ of Gastroenterology, Lian Shui County \\ People's Hospital, Huaian, Jiangsu 223400 , \\ People's Republic of China; ${ }^{5}$ Department \\ of Pathology, Lin Yi Central Hospital, \\ Linyi, Shandong 276400, People's \\ Republic of China
}

Background and objective: Cholangiocarcinoma (CCA) is a highly aggressive neoplasm featured with regional invasiveness and distant metastasis, which often present a phenotype of epithelial-mesenchymal transition (EMT). Long non-coding RNAs (LncRNAs) are dysregulated during carcinogenesis, and up-regulated LncRNA-activated by TGF- $\beta$ (Lnc-ATB) supports tumor growth and metastasis via tumor suppressor microRNA 200 (miR-200). However, the role of Lnc-ATB in CCA is unclear.

Methods: CCA tissues and non-cancer tissues $(n=30)$ were used to determine the Lnc-ATB and $\mathrm{miR}-200 \mathrm{a} / \mathrm{b} / \mathrm{c}$ levels. The functions and mechanisms of Lnc-ATB/miR-200 pathway were determined by knockdown of Lnc-ATB via siRNAs in vitro and in vivo.

Results: CCA tissues have increased Lnc-ATB and reduced miR-200a/b/c levels, but the down-regulated miR-200c was most prominent. Up-regulated Lnc-ATB significant negatively correlated with miR-200c and predicted advanced TNM stage and more lymph node metastasis of CCA patients. Knockdown of Lnc-ATB in two CCA cell lines HuCCT1 and RBE increased miR-200c levels. The luciferase reporter assay further confirmed the direct binding site of miR-200c in Lnc-ATB. Inhibition of Lnc-ATB significantly impaired cell vitality and induced apoptosis and G0/G1 arrest, which, however, was rescued by miR-200c inhibitor. The ability of migration of CCA cells was also up-regulated by Lnc-ATB but was suppressed by miR-200c. Mechanistically, the cell cycle-related CCND1/CDK2, apoptosisrelated BCL-2/caspase-3 and EMT-related E-cadherin/ZEB1/2 were regulated by Lnc-ATB via miR-200c. Knockdown of Lnc-ATB in vivo up-regulated miR-200c signals to inhibit tumor growth with decreased PCNA expression in tumor tissues, which was restored by miR-200c inhibition.

Conclusion: Overexpressed Lnc-ATB functioned as an oncogene for CCA growth and metastasis via miR-200 signals.

Keywords: Lnc-ATB, miR-200, growth, EMT, cholangiocarcinoma

\section{Introduction}

As the second most common primary hepatic tumor, cholangiocarcinoma (CCA) accounts for up to $15 \%$ of all primary liver cancers and its incidence and mortality rates have risen steeply and steadily across the world, the highest rates have been reported in Thailand, China, and other parts of Asia., ${ }^{1,2}$ CA are generally categorized as either intrahepatic or extrahepatic based on their anatomic location with respect to the secondorder bile ducts. ${ }^{3}$ Patients with CCA display a poor prognosis due to its late diagnosis and lack of effective non-surgical curative therapies, which leads to local clearance, vascular
Correspondence: Weichang Chen Department of Gastroenterology, The First Affiliated Hospital of Soochow University, 188 Shizi Street, Suzhou, Jiangsu 215006, People's Republic of China

Tel +86 I 5094769153

Email weichangchen@I26.com 
invasion and lymph node metastases (LNMs). Thus, the 5-year survival rate of patients characterized by a high metastatic potential is only $20-30 \%$. ${ }^{4,5}$ Unfortunately, the exact molecular mechanisms of CCA are not well understood.

Epithelial-to-mesenchymal transition (EMT) is a reversible dynamic process during which epithelial cells gradually adopt structural and functional characteristics of mesenchymal cells. This process involves with the downregulation of epithelial-specific marker E-cadherin via transcription factor Slug, Snail2, ZEB1 and ZEB2, and the up-regulation of mesenchymal markers including Vimentin, and N-cadherin. ${ }^{6,7}$ During carcinogenesis, reduction of E-cadherin was observed in 16.5-82.1\% of CCA patients, which correlated with poor tumor differentiation, tumor size, advanced pTNM stage, intrahepatic metastasis and LNM. $^{8}$ Increasing N-cadherin expression has been associated with lower OS in both CCA subclasses. ${ }^{9}$ However, the mechanism of EMT-mediated metastasis in CCA needs to be further investigated.

Non-coding RNAs (ncRNAs) are defined as RNA transcripts with no protein-coding capacity, which consist of microRNA (miRNA), LncRNA and circle RNA, etc. Accumulating studies in cancer indicated that LncRNAs are key regulators of target gene expression via interacting with miRNA, protein, etc., in various biological processes, such as cell cycle, apoptosis, proliferation, metabolism, and EMT. ${ }^{10,11}$ Many LncRNAs have been reported to be oncogene (e.g., ANRIL, MALAT1, H19, Lnc-CAF, HOTAIR) or tumor suppressor (e.g., MEG3, LincRNA-p21, CASC15-S, Xist). ${ }^{12}$ For example, the LncRNA MEG3 is negatively associated with tumorigenesis and down-regulated in human glioma and esophageal cancer. ${ }^{13,14}$ MEG3 reduces murine double minute 2 homolog (MDM2) expression, causes p53 accumulation, and induces glioma cell growth inhibition and apoptosis in p53dependent and -independent manners. LncRNA that activated by TGF- $\beta$ (Lnc-ATB) can induce ZEB1/2 expressions for EMT initiation by competitively binding miR-200 family and function as an oncogene for tumor growth and metastasis in several types of cancer including colorectal cancer, non-small cell lung cancer, pancreatic cancer, prostate carcinoma, hepatocellular carcinoma, etc. ${ }^{15}$

The tumor cells of CCA patients often present an EMT phenotype and are vulnerable to metastasis. ${ }^{16}$ Thus, we here aimed to investigate the function and clinical significance of Lnc-ATB in CCA and studied the downstream miR-200 signals in vitro and in vivo. The results demonstrated that knockdown of Lnc-ATB signal in CCA cells is promising strategy for the alternative treatment of $\mathrm{CCA}$ patients.

\section{Materials and methods}

\section{Ethics and tissue collection}

All methods used for this study were in accordance with the Ethics Committee of Lin Yi Central Hospital, and this study was conducted in accordance with the Declaration of Helsinki. CCA tissues and corresponding non-cancer tissues $(>1 \mathrm{~cm}$ from the edge of the tumor tissues) were collected in this study $(n=30)$. All patients were pathologically and clinically diagnosed as CCA. All patients completed informed consent forms were also obtained from Lin Yi Central Hospital. None of these cases received chemo-therapy or radiotherapy before surgery.

\section{Cell culture and reagents}

Human bronchial epithelial cell BEC and four CCA cell lines HUCCT1, RBE, TFK1 and Huh-28 were obtained from the American Type Culture Collection (ATCC, Manassas, VA, USA) and cultured in DMEM supplement with 10\% FBS (GIBCO, Waltham, MA, USA) and 1\% penicillin/streptomycin. All the cells were cultured at $37^{\circ} \mathrm{C}$ water-saturated $5 \%$ $\mathrm{CO}_{2}$ atmosphere.

The oligonucleotide sequences of miR-200 mimics, inhibitor or negative control were purchased from GenePharma (Shanghai, China). siRNA-Lnc-ATB or negative control were conducted by RiboBio (Guangzhou, China). psiCHECK2-UTR (wild-type or mutant miR-200c target site) of Lnc-ATB was conducted by GenePharma. The lentivirus vector of siRNA-Lnc-ATB, miR-200c inhibitor or negative control was conducted by GeneChem (Shanghai, China). The antibody to CCND1 (\#ab16663), CDK2 (\#ab32147), and PCNA (\#ab92552) was obtained from Abcam (Massachusetts, USA), and caspase-3 (\#9664), BCL-2 (\#9662), ZEB1/2, E-cadherin and N-cadherin (EMT antibody sampler Kit \#9782) were obtained from Cell Signaling Technology (Denver, MA, USA).

\section{RNA isolation and qRT-PCR}

The total RNA extraction was using Trizol reagent (Invitrogen, CA, USA) which according to the manufacturer's instructions. Reverse transcription reaction was performed using the transcriptase cDNA synthesis kit (Takara, Dalian, China) according to the manufacturer's instructions. Real-time PCR analysis was performed by SYBR Green (Takara) in an ABI 7500 fast fluorescence temperature cycler 
(Waltham, MA, USA). Relative quantification of the target genes including Lnc-ATB, miR-200a/b/c was conducted by the comparative cycle threshold (CT) method $2^{-\Delta \Delta \mathrm{Ct}}$. The expression of GAPDH and U6 was used as the endogenous control. The primer of genes in this study was list in Table 1.

\section{Cell transfection}

The HUCCT1 and RBE cell lines were cultured to about $60 \%$ confluence in 12/96-well plates for the indicated time. The transfections of siRNA-Lnc-ATB or miR-200c inhibitors or negative control were performed by Lipofectamine 2000 (Invitrogen, USA) according to the manufacturer's instructions. After transfection for the indicated time, the cells were harvested for further experiments.

\section{Dual-luciferase reporter assay}

The HUCCT1 cell line was transfected of psiCHECK2UTR (wild-type or mutant miR-200c target site) of LncATB or miR-200c mimics. The luciferase activity assays were performed $24 \mathrm{~h}$ after transfection using a Dual Luciferase Reporter Gene Assay Kit (Beyotime Institute of Biotechnology, Jiangsu, China) according to the manufacturer's protocol. The relative luciferase activity was standardized with renilla luciferase activity.

\section{Cell proliferation, cell cycle and cell apoptosis assays}

The proliferation of HUCCT1 and RBE cells was detected by the Cell Counting Kit-8 (CCK-8; Beyotime Institute of Biotechnology). Cells were dived into Negative control

Table I The primers of genes

\begin{tabular}{|l|l|}
\hline Gene & Primer 5'-3' $^{\prime}$ \\
\hline Lnc-ATB & $\begin{array}{l}\text { F: TCTGGCTGAGGCTGGTTGAC } \\
\text { R: ATCTCTGGGTGCTGGTGAAGG }\end{array}$ \\
\hline GAPDH & $\begin{array}{l}\text { F: ACACCCACTCCTCCACCTTT } \\
\text { R: TTACTCCTTGGAGGCCATGT }\end{array}$ \\
\hline miR-200a & $\begin{array}{l}\text { F: CCTACGCCACAATTAACAAGCC } \\
\text { R: GCCGTCTAACACTGTCTGGTA }\end{array}$ \\
\hline miR-200c & $\begin{array}{l}\text { F: ACACTCCAGCTGGGTAGCACCATTTGA } \\
\text { R: TGGTGTCGTGGAGTCG }\end{array}$ \\
\hline U6 & $\begin{array}{l}\text { F: CCCTCGTCTTACCCAGCAGT } \\
\text { R: CCATCATTACCCGGCAGTAT }\end{array}$ \\
\hline
\end{tabular}

group, siRNA-Lnc-ATB group and siRNA-Lnc-ATB + miR200c inhibitor group. All the cells were seeded in 96well plates, and assayed at 24 and 48 hrs using CCK- 8 kit according to the manufacturer's instructions. Cell viability was assessed by measurement of absorbance at $450 \mathrm{~nm}$ using a microplate reader. Cell apoptosis and cell cycle were analyzed by flow cytometry. The cells were harvested and washed by PBS. $2 \mu \mathrm{L}$ of annexin $\mathrm{V}$ mixed with $2 \mu \mathrm{L}$ of propidium iodide (PI, eBioscience, Waltham, MA, USA) were used to stain cells at $4^{\circ} \mathrm{C}$ for 30 mins for apoptosis analysis. Alternatively, the cells were stained with PI staining solution $\left(10 \mu \mathrm{g} / \mathrm{mL}\right.$ RNase A and $50 \mu \mathrm{g} / \mathrm{mL}$ PI) at $4^{\circ} \mathrm{C}$ for 30 mins in dark and the results were analyzed using a flow cytometry provided with the Cell-Quest software (BD Biosciences, Franklin Lakes, NJ, USA).

\section{Estimation of cell migration}

The Corning Polycarbonate Membrane Insert transwell chambers (Product \#3422; Corning Costar Corp, Cambridge, MA, USA) was used to perform migration assays. Briefly, conditional HUCCT1 and RBE cells $\left(1 \times 10^{5}\right)$ in serum-free media were placed into the upper chamber and medium containing $10 \%$ FBS functioning as a chemo-attractant was placed in the lower chamber. After incubation, cells were fixed with $4 \%$ polyoxymethylene and stained with $1.5 \%$ methylene blue. Cells that did not migrate or invade were removed using a cotton swab and were stained by crystal violet and counted under an inverted microscope. Five random views were selected to count the cells.

\section{Western blot}

After the treatments of cells with the conditions described, ${ }^{17}$ the cells were harvested and the total protein was extracted by $1 \times$ RIPA buffer (Cell Signaling Technology) supplemented with protease inhibitors. The total proteins were separated and analyzed as previously described.

\section{Immunohistochemical analysis}

Immunohistochemistry assays of PCNA were performed as previously described. ${ }^{18}$ Briefly, the frozen sections of tumor tissues were collected and covered with a depth of 2-3 mm with 4\% formaldehyde for 15 mins and washed with PBS. Then, cells were permeabilized with $100 \%$ methanol for 10 mins at $-20^{\circ} \mathrm{C}$ and blocked with $3 \%$ BSA and were incubated with primary antibodies overnight at $4^{\circ} \mathrm{C}$. After rinsing three times in PBS, incubated coverslips in secondary antibody (dilution: 1:400) for $1-2 \mathrm{~h}$ at room temperature. Then, the EnVision Detection System kit (DAKO, 

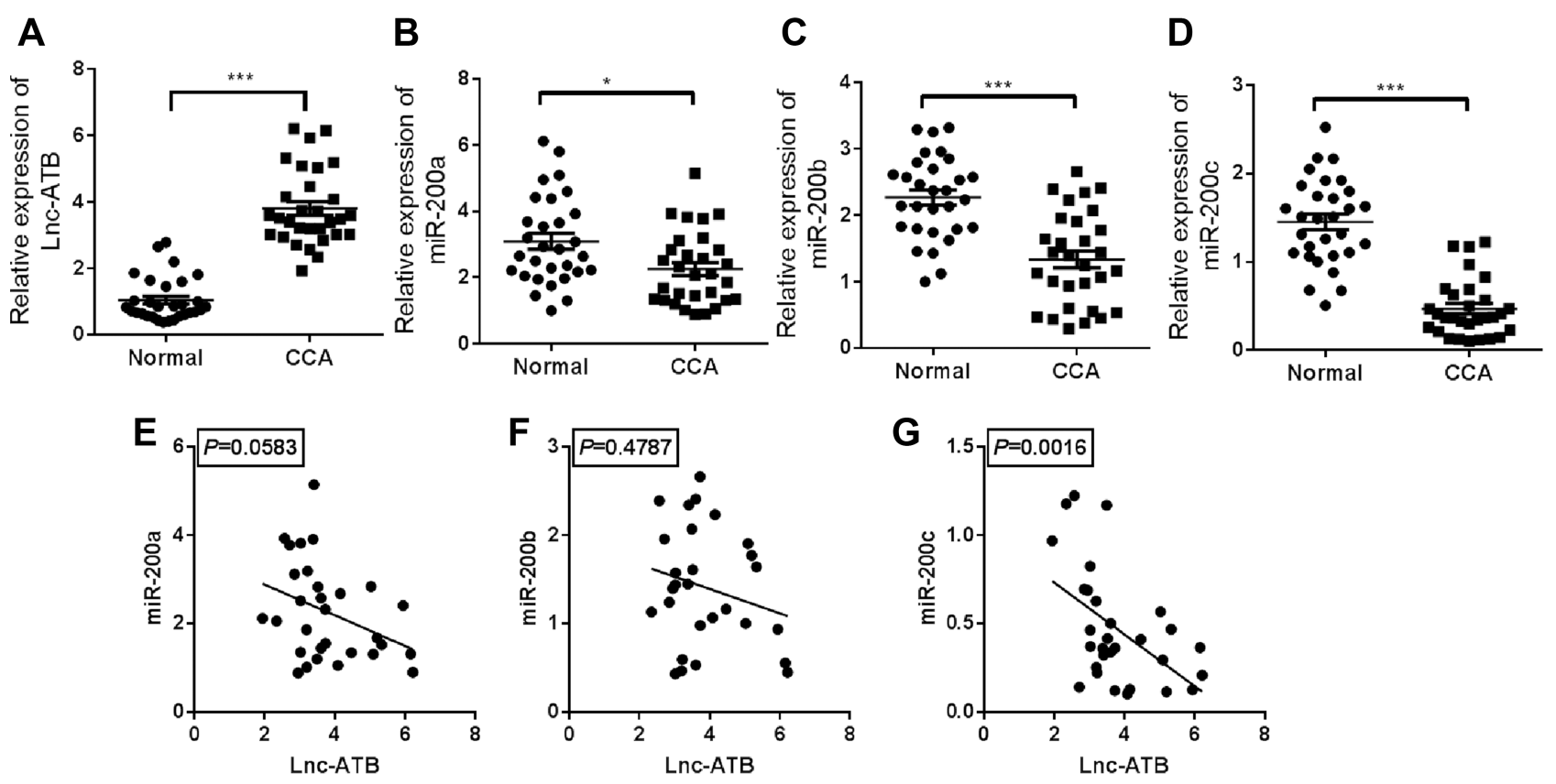

Figure I Increased Lnc-ATB level correlated reduced miR-200c in CCA tissues. (A-D) The expressions of Lnc-ATB and miR-200a/b/c in CCA tissues and non-cancer tissues were determined by Q-PCR. (E-G) The correlations between miR-200 and Lnc-ATB were analyzed. $* P<0.05, * * * P<0.00 \mathrm{I}$, data represent the mean \pm SD. Abbreviation: CCA, cholangiocarcinoma.

Glostrup, Denmark) was used for the DAB chromogen followed by nuclear staining using hematoxylin.

\section{Tumor model}

BALB/c nude mice (age 4 weeks) was purchased from Laboratory Animal Center of Chinese Academy of Sciences (Shanghai, China). The HUCCT1 cells were infected with lentivirus-RNAi-Lnc-ATB (MOI=100), miR-200c inhibitor or negative control. These conditional cells were subcutaneously co-injected in the rear flank of nude mice (5 per group) as indicated description. Results are presented as the mean tumor size $\left(\mathrm{mm}^{3}\right)\left(\mathrm{V}\left(\mathrm{cm}^{3}\right)=\right.$ width ${ }^{2}\left(\mathrm{~cm}^{2}\right) \times$ length $\left.(\mathrm{cm}) / 2\right)$ for every group at various time points until the end-point of the experiment. Animal studies were conducted in accordance with the National Institute of Health Guide for the Care and Use of Laboratory Animals and were approved by the animal ethics committee of Lin Yi Central Hospital.

\section{Statistical analysis}

The data shown in this study were expressed as mean \pm SD. Statistical analyses were performed using MannWhitney tests, the Student's $t$-test and Graph-Pad Prism 5.0 software (GraphPad Software Inc, La Jolla, CA, USA). We used Quantity One and ANOVA to analysis the Western blot band. Data were obtained from three independent experiments and difference significance between groups was assessed as $* P<0.05$; $* * P<0.01$; $* * * P<0.001$.

\section{Results}

\section{The expression of Lnc-Atb is elevated during the development of CCA with decreased miR-200c}

To evaluate the role of Lnc-ATB in CCA, we first estimated the expression of Lnc-ATB and $\mathrm{miR}-200 \mathrm{a} / \mathrm{b} / \mathrm{c}$ in CCA tissues and corresponding non-cancer tissues $(\mathrm{n}=30)$. The Q-PCR results showed that the expression of Lnc-ATB was significantly up-regulated in CCA tissues when compared with non-cancer tissues (Figure 1A). Both the $\mathrm{miR}-200 \mathrm{a} / \mathrm{b} / \mathrm{c}$ were down-regulated in CCA tissues but miR-200c showed to be the most down-regulated miRNA (Figure 1B-D). Besides, the correlations between LncATB and miR-200a/b/c were analyzed and found that upregulated Lnc-ATB significantly correlated miR-200c in CCA with minimal $P$-value (Figure $1 \mathrm{E}-\mathrm{G}$ ). So, we selected Lnc-ATB/miR-200c for further study.

The clinical significances of Lnc-ATB/miR-200c were analyzed. The results indicated that CCA patients with high expression of Lnc-ATB presented high TNM 

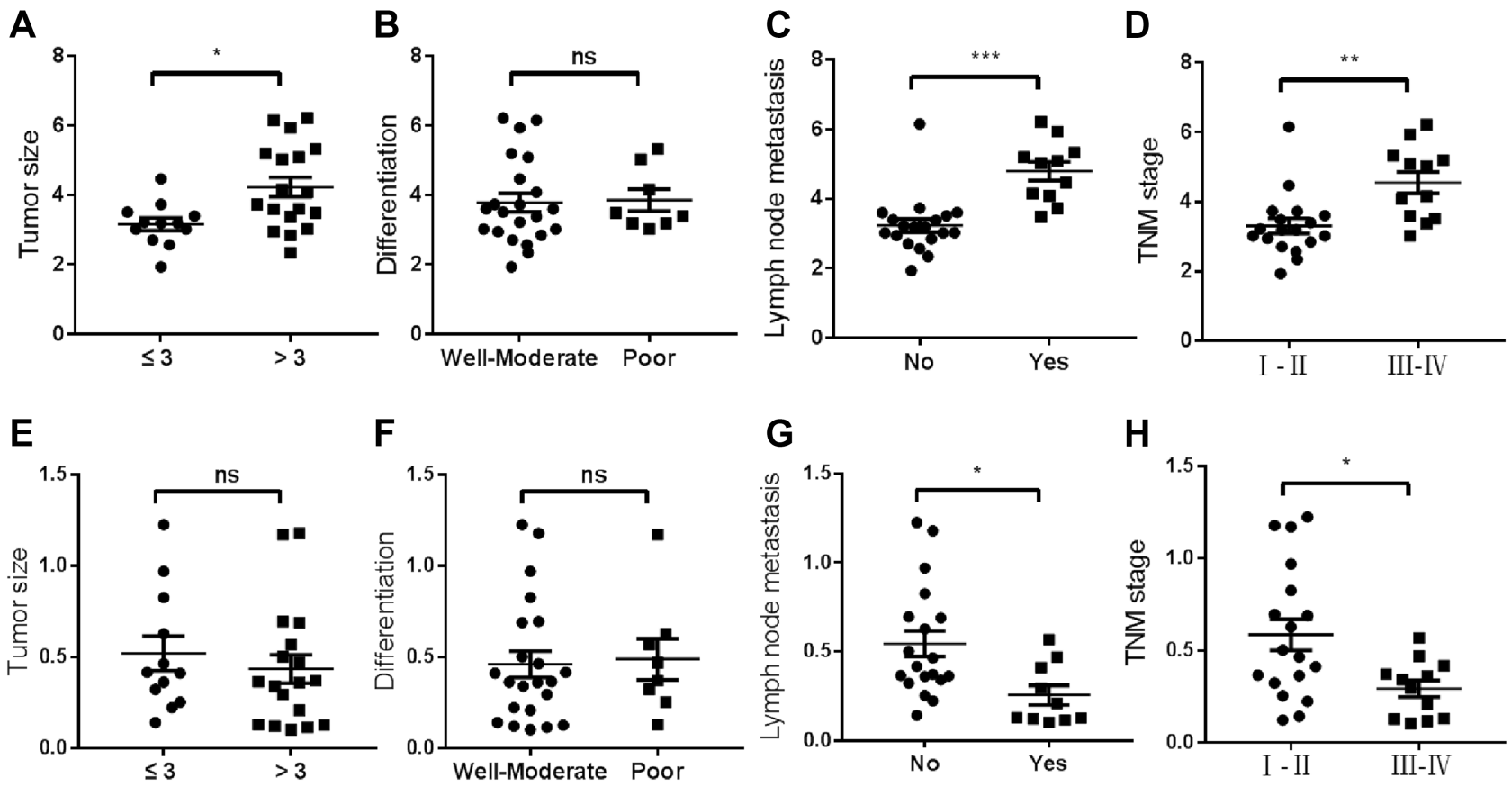

Figure 2 Lnc-ATB/miR-200c predict poor clinical outcome of CCA patients. (A-D) The expressions of Lnc-ATB and (E-H) miR-200c were analyzed in patients with different tumor sizes, differentiation, LNM and TNM stage. $* P<0.05$, $* * P<0.01$, $* * * P<0.001$, data represent the mean \pm SD.

Abbreviations: CCA, cholangiocarcinoma; LNM, lymph node metastasis.

stage, LNM and tumor size (Figure 2A-D). Conversely, low expression of miR-200c predicted poor clinical outcome of CCA patients with high TNM stage and LNM, indicating that Lnc-ATB might function as oncogene and miR-200c function as tumor suppressor in CCA (Figure 2E-H).

\section{Lnc-ATB knockdown up-regulated miR- 200c expression in CCA cells}

We next investigated the relationship of Lnc-ATB/ miR-200c in CCA cells. Human bronchial epithelial cell BEC and four CCA cell lines HUCCT1, RBE, TFK1 and Huh-28 were collected. The Q-PCR results indicated that high expression of Lnc-ATB and low expression of miR-200c were found in CCA cell lines (Figure 3A). SiRNA assay demonstrated that knockdown of Lnc-ATB in HUCCT1 and RBE cells increased the expression of miR-200c (Figure 3B-C). Bioinformatics analysis indicated that Lnc-ATB harbored the potential miR-200c binding site, and the luciferase reporter assay demonstrated that miR-200c mimics significantly impaired the luciferase activity of psiCHECK2-Lnc-ATB with wild miR-200c binding sites, which was not observed in mutant-type (Figure 3D-E). These results indicated that miR-200c could interact with Lnc-ATB in CCA cells.

\section{The availability of miR-200c is antagonized by Lnc-ATB for tumor growth and migration} in vitro

The function of Lnc-ATB/miR-200c in CCA cells was analyzed. Knockdown of Lnc-ATB in HUCCT1 and RBE cells increased the expression of miR-200c (Figure 4A and B), which led to decreased cell vitality in time-dependent manner (Figure $4 \mathrm{C}$ and $\mathrm{D}$ ) and promoted the apoptosis (Figure 4E-F) and G0/G1 arrest (Figure 4G-H). However, when the miR-200c was inhibited by its inhibitor, these processes were restored, indicated that Lnc-ATB was able to participate the cell proliferation and apoptosis via miR-200c in CCA cells.

In addition, the migration of CCA cells was also analyzed by Transwell assay (Figure 5A and B). We found that knockdown of Lnc-ATB inhibited the ability of migration of HUCCT1 and RBE cells which was restored by miR-200c inhibitor via reducing the miR-200c levels (Figure 5C and D).

\section{The pathways involved in Lnc-ATB/ miR-200c axis in CCA}

Since many studies have found that Lnc-ATB/miR-200c signal involved into the proliferation, apoptosis and 

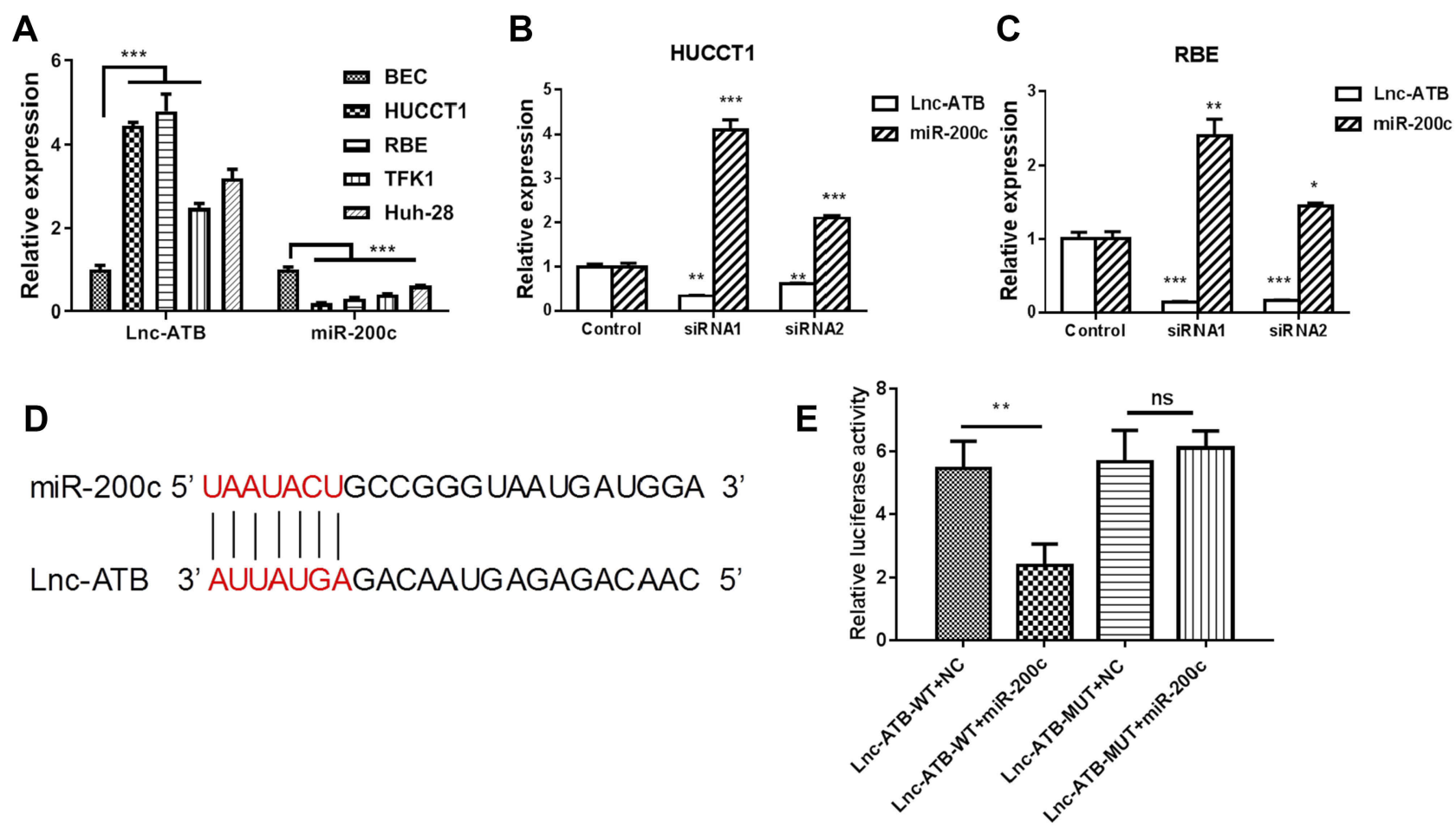

Figure 3 Lnc-ATB interacts with miR-200c in CCA cells. (A) The expression of Lnc-ATB and miR-200c in CCA cell lines was estimated by Q-PCR. (B, C) Knockdown of Lnc-ATB in HUCCTI and RBE cells by siRNA and the expression of Lnc-ATB and miR-200c were determined by Q-PCR. (D) The target site of miR-200c to Lnc-ATB in CCA. (E) The HUCCTI cells were transfected with wild or mutant $p s i C H E C K 2$-Lnc-ATB with wild miR-200c mimics to determine the luciferase activity. $* P<0.05$, $* * P<0.01$, $* * * P<0.001$, data represent the mean \pm SD.

Abbreviation: CCA, cholangiocarcinoma.

metastasis pathways in tumor cells, ${ }^{15}$ we here investigated the cell cycle-related, apoptosis-related and EMT-related pathways in HUCCT1 cells. The results showed that the expressions of CCND1 and CDK2 were inhibited by Lnc-ATB knockdown (Figure 6A). The anti-apoptosis BCL-2 was down-regulated by Lnc-ATB knockdown with increased cleaved caspase-3, which was restored by miR-200c inhibitor (Figure 6B). ZEB1/2 was the target genes of miR-200c for EMT phenotype. Inhibition of LncATB could up-regulate the miR-200c expression to inhibit the expression of ZEB1/2, leading to enhancing the expressions of epithelial-specific marker E-cadherin and reduce the mesenchymal markers $\mathrm{N}$-cadherin (Figure 6C).

\section{Lnc-ATB is required for CCA growth via regulation of miR-200c in vivo}

The xenograft model of conditional HUCCT1 cells was established and HUCCT1 cells were treated with lentivirussiRNA-Lnc-ATB and/or miR-200c inhibitor (Figure 7A and C). The results showed that mice with siRNA-Lnc-ATB had less tumor burden with less expression of PCNA in tumor tissues than the mice with siRNA-control. However, the treatment of miR-200c inhibitor abrogated Lnc-ATB knockdown-induced tumor regression and restored the expression of PCNA (Figure 7B and D). Meanwhile, the cell cyclerelated, apoptosis-related and EMT-related pathways were analyzed in tumor tissues. CCND1/CDK2 was positively regulated by Lnc-ATB via miR-200c. Cleaved caspase-3 was up-regulated in tumors with siRNA-Lnc-ATB and BCL-2was increased in siRNA-Lnc-ATB/miR-200c inhibitor. The EMT phenotype was also induced by Lnc-ATB and impaired by miR-200c in tumor tissues (Figure 7E).

\section{Discussion}

Surgical resection is the only curative treatment, but it is only available for a small percentage of patients with early-stage disease, and only $20-30 \%$ of these patients survive after 5 years due to the high rate of recurrence and metastasis after surgery. ${ }^{19}$ Therefore, the investigations on identifying potential therapeutic targets for alternative strategy are necessary for the treatment of CCA. In this study, we firstly found that Lnc-ATB functioned as oncogene to promote CCA growth and migration via regulation of miR-200 signals. 


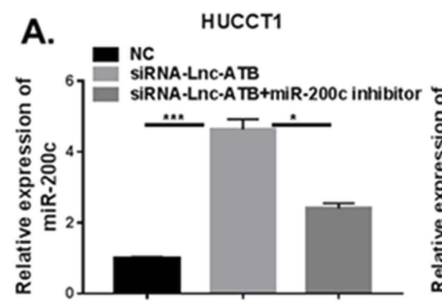

B.

RBE

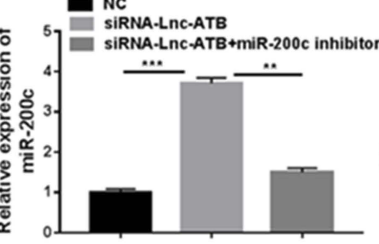

E.

F.
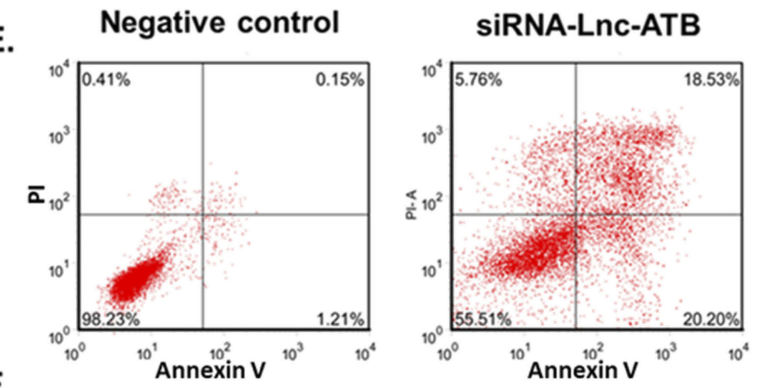

C.
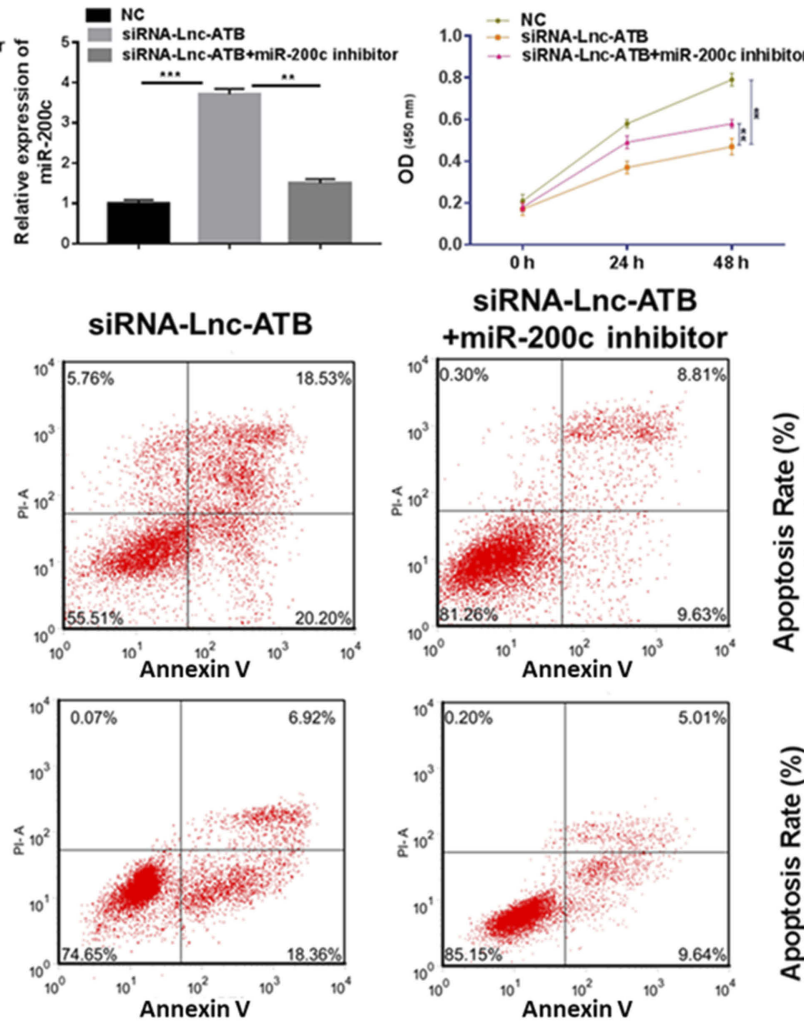

SiRNA-Lnc-ATB

G.

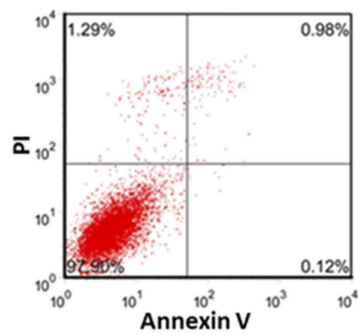

Negative control
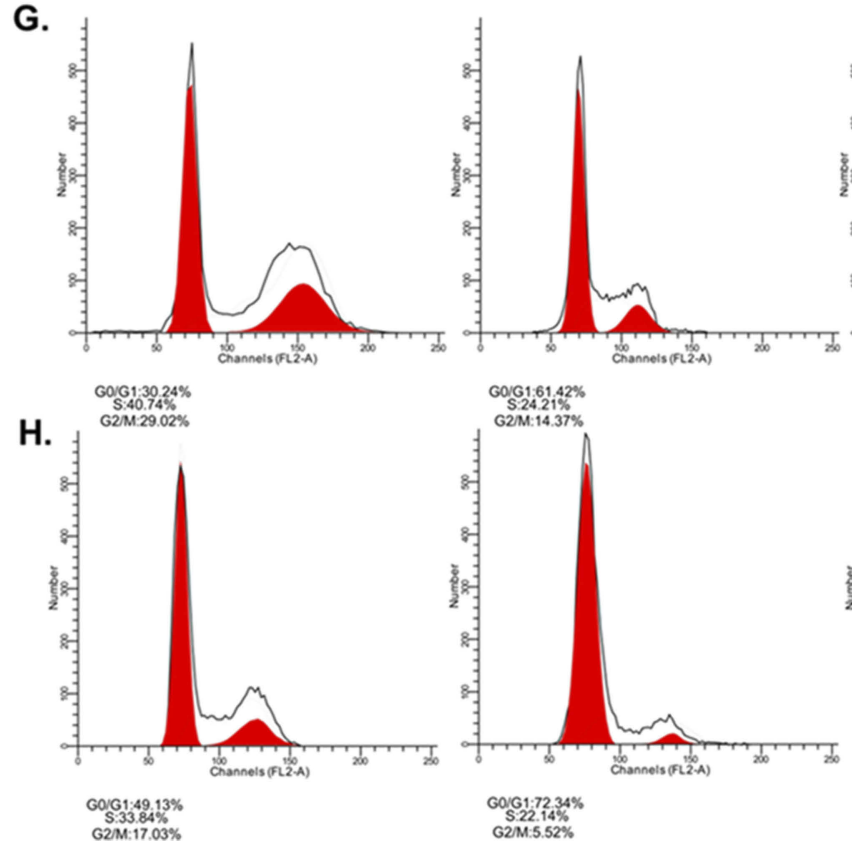

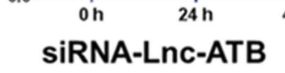

+miR-200c inhibitor

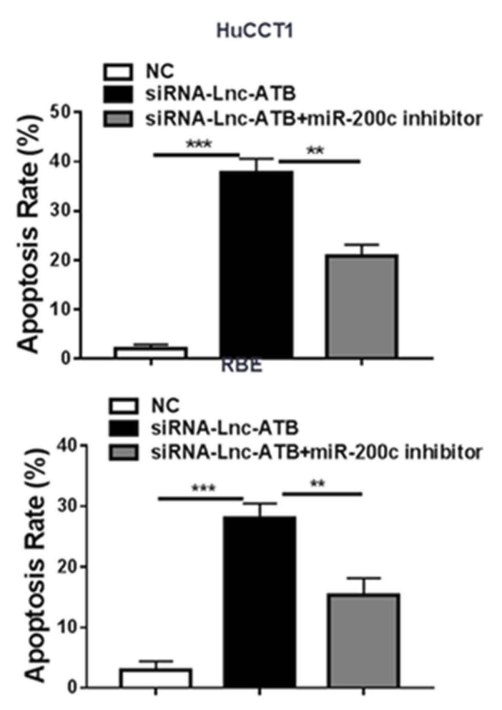

D. $\mathrm{RBE}$

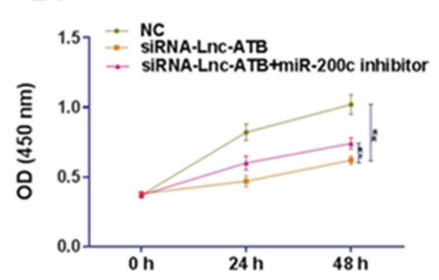

+miR-200c inhibitor
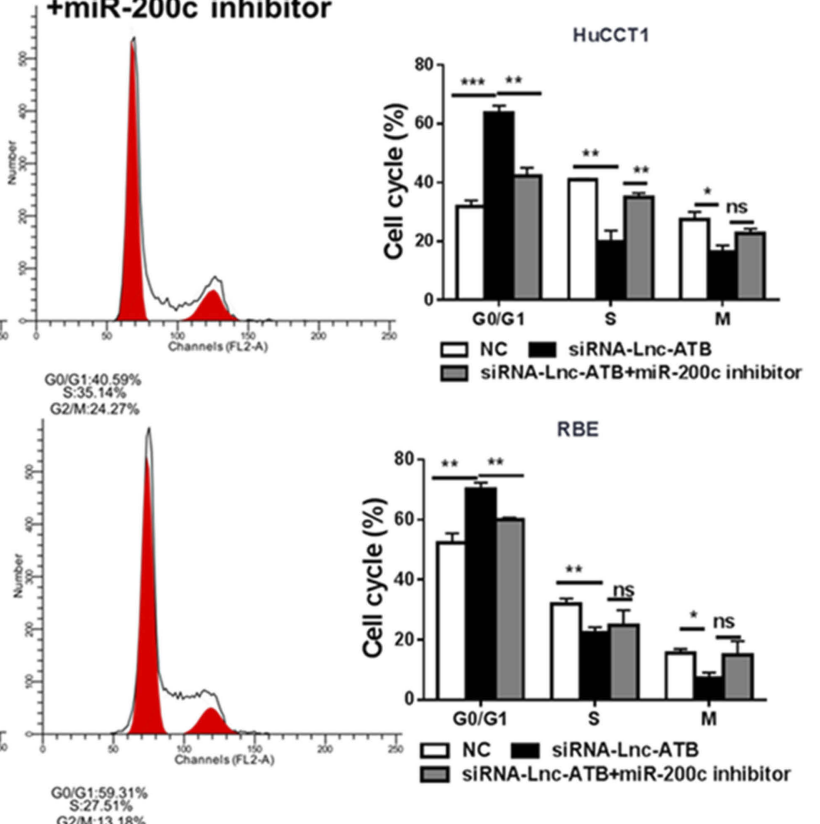

Figure 4 Knockdown of Lnc-ATB inhibits CCA cell growth in vitro. (A, B) The HUCCTI and RBE cells were transfected with siRNA-Lnc-ATB and miR-200c inhibitor, and the expressions of Lnc-ATB and miR-200c were determined. Besides, (C, D) the cell vitalities were determined by CCK-8 assay, (E, F) the apoptosis and (G, H) cell cycle was analyzed by flow cytometry. $* P<0.05, * * P<0.0 \mathrm{I}$, $* * * P<0.00 \mathrm{I}$, data represent the mean $\pm \mathrm{SD}$. The $\mathrm{X}$-aixs of scatter plots in Figure $4 \mathrm{E} / \mathrm{F}$ is "Annexin $\mathrm{V}$ " and the $\mathrm{Y}$-aixs of scatter plots in Figure 4E/F is "PI".

Abbreviations: CCA, cholangiocarcinoma; CCK-8, Cell Counting Kit-8.

Actually, there have been some studies identified the LncRNA CCAT1 is overexpressed in CCA tissues and potential target therapeutic role of LncRNAs in CCA. predicted more LNM and more advanced tumor stage 


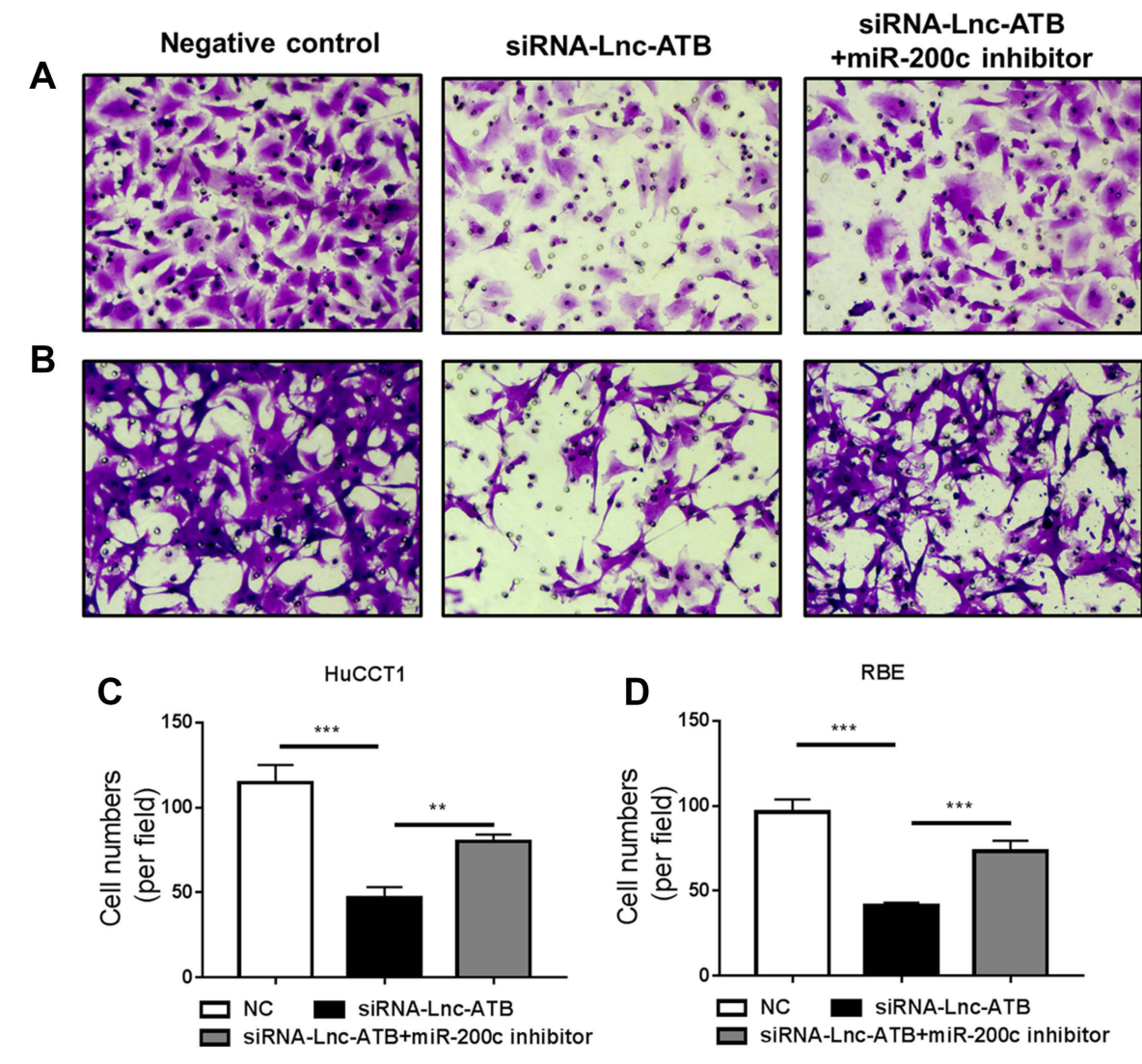

Figure 5 Knockdown of Lnc-ATB inhibits CCA cell migration in vitro. The HUCCTI and RBE cells were transfected with siRNA-Lnc-ATB and miR-200c inhibitor, the migration of $(\mathbf{A}$ and $\mathbf{C}) \mathrm{HUCCTI}$ and $(\mathbf{B}$ and $\mathbf{D}) \mathrm{RBE}$ cells were analyzed by Transwell assay. Five random views were selected to count the cells. $* * P<0.0 \mathrm{I}$, $* * * P<0.00 \mathrm{I}$, data represent the mean \pm SD.

Abbreviations: CCA, cholangiocarcinoma; NC, normal control.

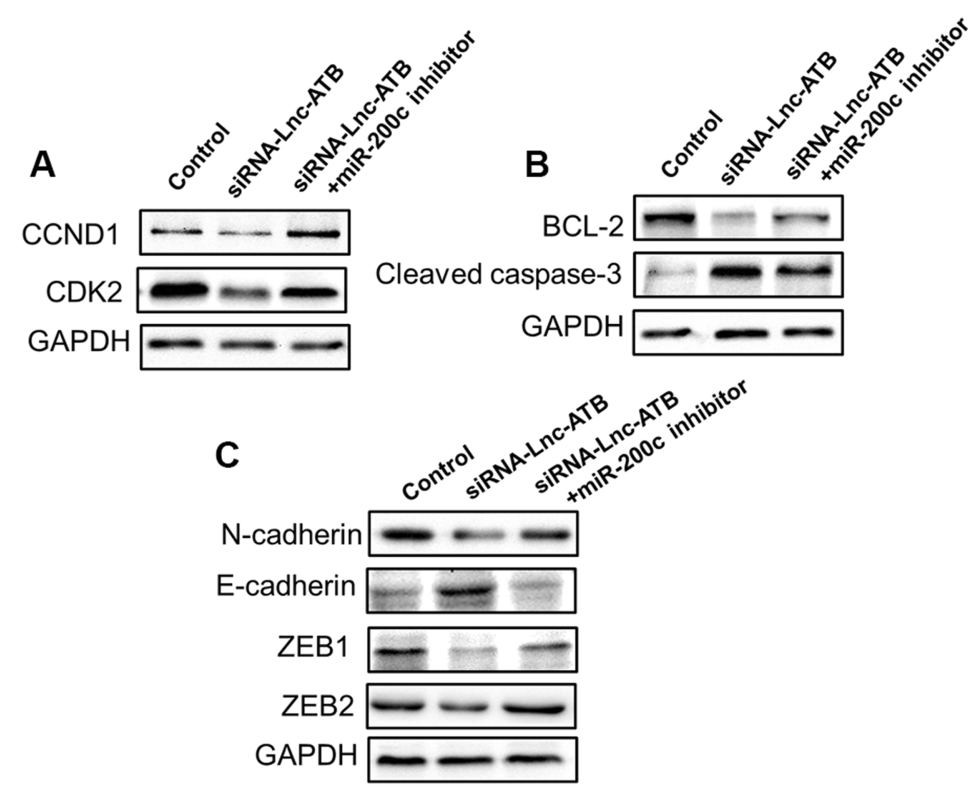

Figure 6 The pathways involved into Lnc-ATB/miR-200c signals in CCA. The HUCCTI were transfected with siRNA-Lnc-ATB and miR-200c inhibitor, (A) the cell cycle pathway, (B) the apoptosis and (C) the EMT pathway were analyzed by WB in HUCCTI cells.

Abbreviations: CCA, cholangiocarcinoma; EMT, endothelial mesenchymal trasmission WB, Western blot. 


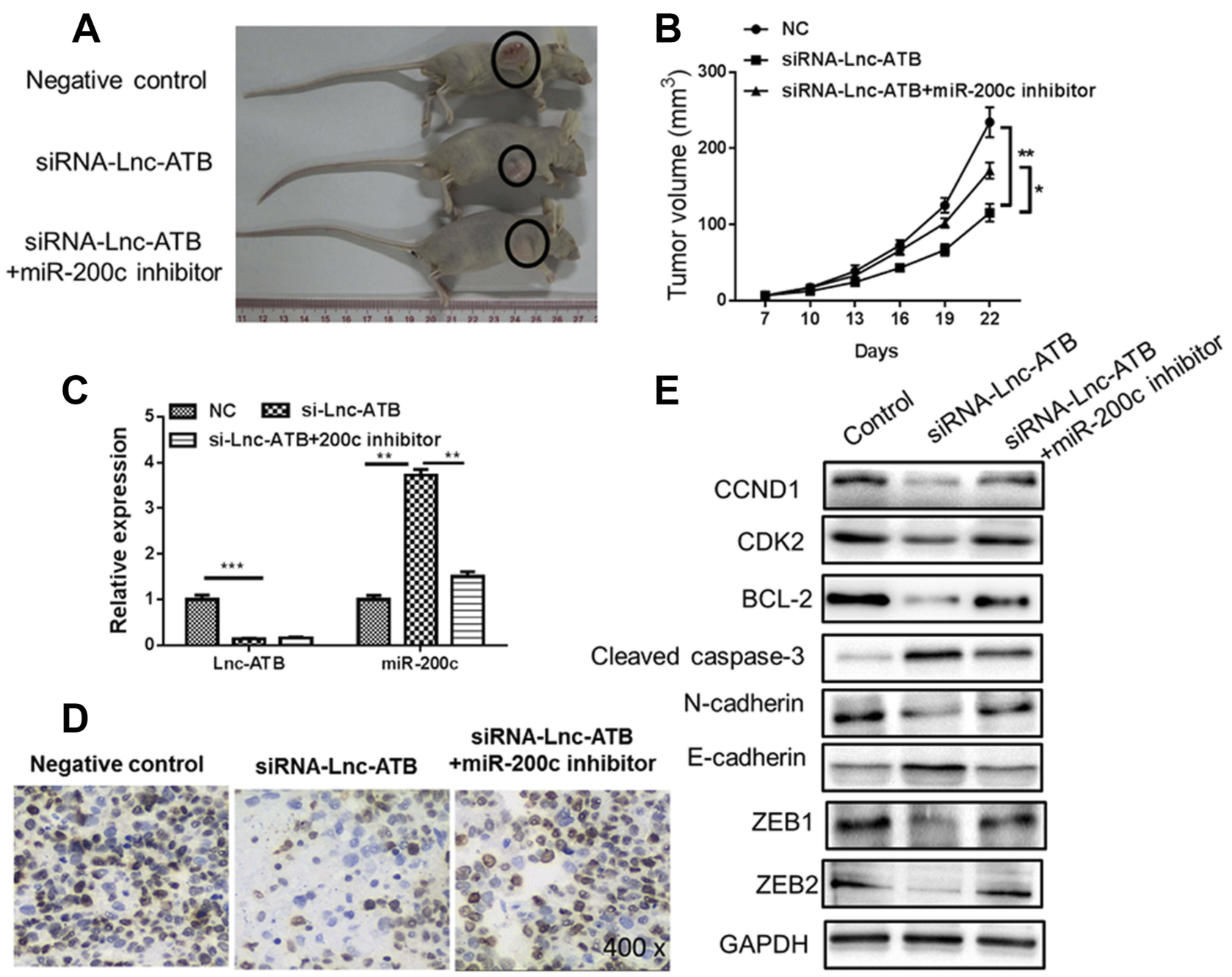

Figure 7 Knockdown of Lnc-ATB induces tumor regression of CCA in vivo. The xenograft model of conditional HUCCTI cells was established (5 per group) and HUCCTI cells were treated with lentivirus-siRNA-Lnc-ATB and/or miR-200c inhibitor. (A, B) The tumor volume was calculated (C) and the expression of Lnc-ATB and miR-200c was analyzed by Q-PCR. (D) The expression of proliferation marker PCNA was analyzed in tumor tissues by IHC analysis. (E) The pathways involved into Lnc-ATB/miR-200c signals were estimated by $W B$ in tumor tissues. $* P<0.05$, $* * P<0.01$, $* * * P<0.001$, data represent the mean $\pm S D$.

Abbreviations: CCA, cholangiocarcinoma; WB, Western blot.

and could be an independent prognostic factor for CCA. ${ }^{20}$ LncRNA Sox2ot was up-regulated in CCA tissues and was significantly associated with lymph node invasion, TNM stage and postoperative recurrence. ${ }^{21}$ Shi et al reported that LncRNA AFAP1-AS1 expression was higher in CCA tumors than matched adjacent non-tumor tissue. ${ }^{22}$ Similarly, $\mathrm{Xu}$ et al found that LncRNA PANDAR was significantly upregulated in CCA tissue specimens and cell lines, and its high expression was closely associated with lymph node invasion, TNM stage and postoperative relapse in patients with $\mathrm{CCA} .{ }^{23}$ We here identified that Lnc-ATB was also functioned as oncogene during the development of CCA. Up-regulated Lnc-ATB correlated with high TNM stage and LNM of CCA patients.
The interaction among mammalian lncRNAs and miRNAs has been the pivotal mechanism of LncRNA-mediated tumor behaviors. LncRNAs are targeted by miRNAs to reduce IncRNA stability. LncRNAs can also function as molecular decoys or sponges of microRNAs to compete with miRNAs for binding to shared target mRNAs. ${ }^{12}$ Wang et al demonstrated that the H19 and HULC levels were elevated under the hypoxic or inflammatory condition. Inhibition of those two IncRNAs in CCA cells suppressed their migration and invasion potential via sponging let-7a/let-7b and miR-372/miR-373 and activating the IL-6, CXCR4 pathway. ${ }^{24}$ MALAT1 promoted the proliferation, migration, and invasion of human CCA cells via miR-204-dependent CXCR4 regulation. ${ }^{25}$ Lnc-ATB is up-regulated by TGF- $\beta$ in many cancers and is physically 
associated with the miR-200 family to up-regulate ZEB1 and ZEB2 levels for EMT phenotype. ${ }^{26}$ Although miR-200 family has been found to be down-regulated in CCA tissues, we found that miR-200c showed the highest correlation with Lnc-ATB in CCA tissues.

Although the function of Lnc-ATB has investigated in many cancers, its role in CCA was firstly studied here. In this study, we found that Lnc-ATB could abrogate the availability of miR-200c in CCA cells. Knockdown of Lnc-ATB inhibited the cell proliferation and migration, induced apoptosis and G0/G1 arrest via up-regulation of miR-200c. In addition, other miRNAs were also reported to be interacted with Lnc-ATB. Cao et al reported that miR-494 was inhibited by Lnc-ATB in lung cancer and Lnc-ATB increased phosphorylated levels of AKT, JAK1, and STAT3, which was reversed by miR-494 overexpression. ${ }^{27}$ Lnc-ATB in gastric cancer also promoted tumor progression by interacting with miR-141-3p to inhibit the expression of TGF- $\beta 2$ and repressed proliferation and induced cell cycle arrest. ${ }^{28} \mathrm{We}$ found that the cell cycleregulator CCND1/CDK2 and the anti-apoptosis BCL-2 were positively regulated by Lnc-ATB/miR-200c for the maintenance of cell cycle and growth. The target genes of miR-200c, ZEB1/2 were also found to be up-regulated by Lnc-ATB in CCA cells. Therefore, Lnc-ATB/miR-200c signals participated into the CCA progression.

Taken together, we here identified that up-regulated Lnc-ATB in CCA cells promoted the cell growth and migration via reducing the expression of tumor suppressor miR-200c. Inhibition of miR-200c abrogated Lnc-ATB knockdown-induced tumor regression in vivo, which involved with cell cycle-related CCND1/CDK2, apoptosis-related BCL-2/caspase-3 and EMT-related E-cadherin/ $\mathrm{N}$-cadherin/ZEB1/2 signals.

\section{Author contributions}

All authors contributed toward data analysis, drafting and revising the paper, gave final approval of the version to be published and agree to be accountable for all aspects of the work.

\section{Disclosure}

The authors report no conflicts of interest in this work.

\section{References}

1. Khan SA, Davidson BR, Goldin RD, et al. Guidelines for the diagnosis and treatment of cholangiocarcinoma: an update. Gut. 2012;61 (12):1657-1669. doi:10.1136/gutjnl-2011-301748
2. Lim JH, Park CK. Pathology of cholangiocarcinoma. Abdom Imaging. 2004;29(5):540-547. doi:10.1007/s00261-004-0187-2

3. Hammill CW, Wong LL. Intrahepatic cholangiocarcinoma: a malignancy of increasing importance. J Am Coll Surg. 2008;207(4):594603. doi:10.1016/j.jamcollsurg.2008.04.031

4. Esnaola NF, Meyer JE, Karachristos A, Maranki JL, Camp ER, Denlinger CS. Evaluation and management of intrahepatic and extrahepatic cholangiocarcinoma. Cancer. 2016;122(9):1349-1369. doi:10.1002/cner.29692

5. Gatto M, Bragazzi MC, Semeraro R, et al. Cholangiocarcinoma: update and future perspectives. Dig Liver Dis. 2010;42(4):253-260. doi:10.1016/j.dld.2009.12.008

6. Santamaria PG, Moreno-Bueno G, Portillo F, Cano A. EMT: present and future in clinical oncology. Mol Oncol. 2017;11(7):718-738. doi:10.1002/1878-0261.12091

7. Singh M, Yelle N, Venugopal C, Singh SK. EMT: mechanisms and therapeutic implications. Pharmacol Ther. 2018;182:80-94. doi:10.1016/j.pharmthera.2017.08.009

8. Mao X, Chen D, Wu J, et al. Differential expression of fascin, Ecadherin and vimentin: proteins associated with survival of cholangiocarcinoma patients. Am J Med Sci. 2013;346(4):261-268. doi:10.1097/MAJ.0b013e3182707108

9. Yu TH, Yuan RH, Chen YL, Yang WC, Hsu HC, Jeng YM. Viral hepatitis is associated with intrahepatic cholangiocarcinoma with cholangiolar differentiation and N-cadherin expression. Mod Pathol. 2011;24(6):810-819. doi:10.1038/modpathol.2011.41

10. Ding L, Ren J, Zhang D, et al. The TLR3 agonist inhibit drug efflux and sequentially consolidates low-dose cisplatin-based chemoimmunotherapy while reducing side effects. Mol Cancer Ther. 2017;16 (6):1068-1079. doi:10.1158/1535-7163.MCT-16-0454

11. Tang Y, Cheung BB, Atmadibrata B, et al. The regulatory role of long noncoding RNAs in cancer. Cancer Lett. 2017;391:12-19. doi:10.1016/j.canlet.2017.01.010

12. Schmitt AM, Chang HY. Long noncoding RNAs in cancer pathways. Cancer Cell. 2016;29(4):452-463. doi:10.1016/j.ccell.2016.03.010

13. Gong X, Huang M. Long non-coding RNA MEG3 promotes the proliferation of glioma cells through targeting Wnt/beta-catenin signal pathway. Cancer Gene Ther. 2017;24(9):381-385. doi:10.1038/cgt.2017.32

14. Dong Z, Zhang A, Liu S, et al. Aberrant methylation-mediated silencing of lncRNA MEG3 functions as a ceRNA in esophageal cancer. Mol Cancer Res. 2017;15(7):800-810. doi:10.1158/15417786.MCR-16-0385

15. Li J, Li Z, Zheng W, et al. LncRNA-ATB: an indispensable cancerrelated long noncoding RNA. Cell Prolif. 2017;50:6. doi:10.1111/ cpr. 12368

16. Vaquero J, Guedj N, Claperon A, Nguyen Ho-Bouldoires TH, Paradis $\mathrm{V}$, Fouassier L. Epithelial-mesenchymal transition in cholangiocarcinoma: from clinical evidence to regulatory networks. $J$ Hepatol. 2017;66(2):424-441. doi:10.1016/j.jhep.2016.09.010

17. $\mathrm{Li} \mathrm{Z}, \mathrm{Wu} \mathrm{X}, \mathrm{Gu} \mathrm{L}$, et al. Long non-coding RNA ATB promotes malignancy of esophageal squamous cell carcinoma by regulating miR-200b/Kindlin-2 axis. Cell Death Dis. 2017;8(6):e2888. doi:10.1038/cddis.2017.245

18. Ding L, Ren J, Zhang D, et al. A novel stromal lncRNA signature reprograms fibroblasts to promote the growth of oral squamous cell carcinoma via LncRNA-CAF/interleukin-33. Carcinogenesis. 2018;39(3):397-406. doi:10.1093/carcin/bgy006

19. Patel T. Cholangiocarcinoma. Nat Clin Pract Gastroenterol Hepatol. 2006;3(1):33-42. doi:10.1038/ncpgasthep0389

20. Jiang XM, Li ZL, Li JL, et al. LncRNA CCAT1 as the unfavorable prognostic biomarker for cholangiocarcinoma. Eur Rev Med Pharmacol Sci. 2017;21(6):1242-1247.

21. Li Z, Li J, Ji D, et al. Overexpressed long noncoding RNA Sox2ot predicts poor prognosis for cholangiocarcinoma and promotes cell proliferation and invasion. Gene. 2018;645:131-136. doi:10.1016/j. gene.2017.12.017 
22. Shi X, Zhang H, Wang M, et al. LncRNA AFAP1-AS1 promotes growth and metastasis of cholangiocarcinoma cells. Oncotarget. 2017;8(35):58394-58404. doi:10.18632/oncotarget.16880

23. Xu Y, Jiang X, Cui Y. Upregulated long noncoding RNA PANDAR predicts an unfavorable prognosis and promotes tumorigenesis in cholangiocarcinoma. Onco Targets Ther. 2017;10:2873-2883. doi:10.2147/ OTT.S137044

24. Wang WT, Ye H, Wei PP, et al. LncRNAs H19 and HULC, activated by oxidative stress, promote cell migration and invasion in cholangiocarcinoma through a ceRNA manner. J Hematol Oncol. 2016;9 (1):117. doi:10.1186/s13045-016-0348-0

25. Tan X, Huang Z, Li X. Long non-coding RNA MALAT1 interacts with miR-204 to modulate human hilar cholangiocarcinoma proliferation, migration, and invasion by targeting CXCR4. J Cell Biochem. 2017;118(11):3643-3653. doi:10.1002/jcb.25862
26. Yuan JH, Yang F, Wang F, et al. A long noncoding RNA activated by TGF-beta promotes the invasion-metastasis cascade in hepatocellular carcinoma. Cancer Cell. 2014;25(5):666-681. doi:10.1016/j.ccr.2014. 03.010

27. Cao Y, Luo X, Ding X, Cui S, Guo C. LncRNA ATB promotes proliferation and metastasis in A549 cells by down-regulation of microRNA-494. J Cell Biochem. 2018;119(8):6935-6942. doi:10.10 02/jcb. 26894

28. Lei K, Liang X, Gao Y, et al. Lnc-ATB contributes to gastric cancer growth through a MiR-141-3p/TGFbeta2 feedback loop. Biochem Biophys Res Commun. 2017;484(3):514-521. doi:10.1016/j.bbrc.20 17.01 .094

\section{Publish your work in this journal}

OncoTargets and Therapy is an international, peer-reviewed, open access journal focusing on the pathological basis of all cancers, potential targets for therapy and treatment protocols employed to improve the management of cancer patients. The journal also focuses on the impact of management programs and new therapeutic

Submit your manuscript here: https://www.dovepress.com/oncotargets-and-therapy-journal agents and protocols on patient perspectives such as quality of life, adherence and satisfaction. The manuscript management system is completely online and includes a very quick and fair peer-review system, which is all easy to use. Visit http://www.dovepress.com/ testimonials.php to read real quotes from published authors. 